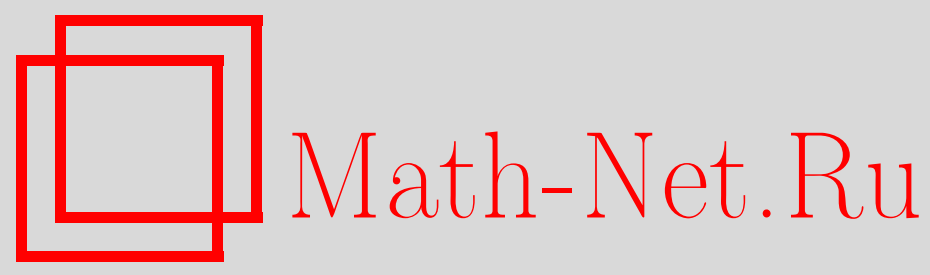

Ю. Н. Фролов, Разложение целых функций в ряд Лагранжа, Матем. заметки, 1997, том 61, выпуск 1, 119-124

DOI: https://doi.org/10.4213/mzm1486

Использование Общероссийского математического портала Math-Net.Ru подразумевает, что вы прочитали и согласны с пользовательским соглашением http://www. mathnet.ru/rus/agreement

Параметры загрузки:

IP: 3.85 .73 .92

26 апреля 2023 г., 15:29:37

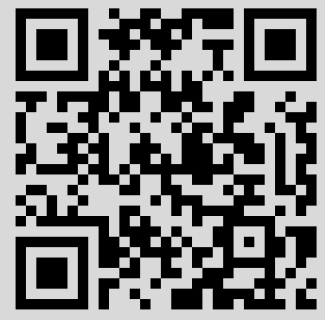




\section{РАЗЛОЖЕНИЕ ЦЕЛЫХ ФУНКЦИЙ В РЯД ЛАГРАНЖА}

\section{Ю.Н. Фролов}

В работе исследуется задача о представимости целых функций пространств, описываемых порядком и типом, рядами Лагранжа, сходящимися в естественной для этих пространств топологии, которая сильее топологии компактной сходимости.

Библиограффия: 3 названия.

1. Постановка задачи. Пусть $L(\lambda)$ - целая функция, имеющая простые нули $\lambda_{1}$, $\lambda_{2}, \ldots$ Произвольной целой функции $M(\lambda)$ формально можно сопоставить ряд

$$
M(\lambda) \sim \sum_{\nu=1}^{\infty} \frac{L(\lambda)}{\lambda-\lambda_{\nu}} \frac{M\left(\lambda_{\nu}\right)}{L^{\prime}\left(\lambda_{\nu}\right)},
$$

который назьвается интерполящионньм рядом Лагранжа функции $M(\lambda)$ с узлами интерполяции $\left\{\lambda_{\nu}\right\}$.

Вопросам разложимости целых функций из того или иного класса в ряд Лагранжа в топологии равномерной сходимости на любом компакте посвящено немалое количество работ. Наиболее важные результаты в этой области приведены, например, в монографии И. И. Ибрагимова [1].

В настоящей работе исследуется вопрос о представимости целых функций пространств, описываемых порядком и типом, рядами Лагранжа, сходящимися в естественной для этих пространств топологии, которая сильнее, нежели топология компактной сходимости.

Дадим краткое описание этих пространств.

Пусть $0<\alpha<\infty$ и $0<\beta<\infty$. Для любого $\varepsilon>0$ через $G_{\varepsilon}$ обозначим банахово пространство целых функций $F(z)$, для которых конечны нормы

$$
\begin{gathered}
\|F\|_{\varepsilon}=\sup _{r}\left\{M_{F}(r) \exp \left[-(\beta+\varepsilon) r^{\alpha}\right]\right\}, \\
M_{F}(r)=\max _{|z|=r}|F(z)| .
\end{gathered}
$$

Проективный предел семейства $\left\{G_{\varepsilon}\right\}$ обозначим через $[\alpha, \beta]$. Это пространство состоит из целых функций порядка меньшего $\alpha$, либо порядка $\alpha$, но типа не вьше $\beta$. Последовательность $\left\{F_{n}(z)\right\}$ сходится к $F(z)$ в $[\alpha, \beta]$ тогда и только тогда, когда

1) $F_{n}(z) \rightarrow F(z)$ равномерно на любом компакте;

2) $\forall \varepsilon>0 \exists r_{0}(\varepsilon)$, не зависящее от $n$, такое, что

$$
\left|F_{n}(z)\right|<\exp \left[(\beta+\varepsilon)|z|^{\alpha}\right], \quad|z|>r_{0}(\varepsilon) .
$$


Далее, для любого $0<h<\beta$ через $N_{h}$ обозначим банахово пространство цельх функций $F(z)$, для которых конечны нормы

$$
\|F\|_{h}=\sup _{r}\left[M_{F}(r) \exp \left(-h r^{\alpha}\right)\right] .
$$

Индуктивньй предел семейства $\left\{N_{h}\right\}$ обозначим через $[\alpha, \beta)$. Пространство $[\alpha, \beta)$ состоит из целых функций порядка либо меньшего $\alpha$, либо равного $\alpha$, но тогда типа меньшего $\beta$. Последовательность $\left\{F_{n}(z)\right\}$ сходится к $F(z)$ в $[\alpha, \beta)$ тогда и только тог да, когда

1) $F_{n}(z) \rightarrow F(z)$ равномерно на любом компакте;

2) существуют $h<\beta$ и $r_{0}(h)$, не зависящие от $n$, такие, что

$$
\left|F_{n}(z)\right|<\exp \left[h|z|^{\alpha}\right], \quad|z|>r_{0}(h) .
$$

Теперь обратимся к задаче.

Будем считать $L(\lambda)$ целой функцией порядка $\rho_{1}>0$, типа $\sigma_{1} \neq 0, \infty, L(0) \neq 0$ и $\left\{\lambda_{\nu}\right\}$ - ее простые нули.

Зададимся вопросом: какими свойствами должна обладать функция $L(\lambda)$, чтобы $\forall M(\lambda) \in\left[\rho_{1}, \sigma_{1}\right)$ разлагалась в топологии $\left[\rho_{1}, \sigma_{1}\right]$ в свой ряд Лагранжа $(1) ?$

Заметим, во-первых, что брать $M(\lambda)$ из более широкого класса, вообще говоря, нельзя. В самом деле, если считать классом разлагаемых функций хотя бы $\left[\rho_{1}, \sigma_{1}\right]$, то $M(\lambda)=\lambda L^{\prime}(\lambda) \in\left[\rho_{1}, \sigma_{1}\right]$ и ее ряд $(1)$

$$
\sum_{\nu=1}^{\infty} \frac{L(\lambda)}{\lambda-\lambda_{\nu}} \frac{\lambda_{\nu} L^{\prime}\left(\lambda_{\nu}\right)}{L^{\prime}\left(\lambda_{\nu}\right)}
$$

при $\lambda=0$ расходится. Таким образом, не любая функций из $\left[\rho_{1}, \sigma_{1}\right]$ разлагается в ряд (1).

Заметим, во-вторых, что усилить топологию, в которой $M(\lambda)$ разлагается в ряд $(1)$, также нельзя. Действительно, пусть $M(\lambda) \in\left[\rho_{1}, \sigma_{1}\right)$ разлагается в топологии $\left[\rho_{1}, \sigma_{1}\right)$ в ряд (1). Тогда имеются $\sigma^{\prime}<\sigma_{1}$ и $C>0$ такие, что при любом $\nu$ и $|\lambda|>r_{0}$, где $r_{0}$ не зависит от $\nu$, справедливо неравенство

$$
\left|\frac{L(\lambda)}{\lambda-\lambda_{\nu}} \frac{M\left(\lambda_{\nu}\right)}{L^{\prime}\left(\lambda_{\nu}\right)}\right|<C \exp \left(\sigma^{\prime}|\lambda|^{\rho_{1}}\right) .
$$

Поскольку $L(\lambda)$ - целая функция типа $\sigma_{1}$ при порядке $\rho_{1}$, для любого $\sigma^{\prime \prime}<\sigma_{1}$ можно указать последовательность уходящих на бесконечность чисел $\left\{\alpha_{k}\right\}$ таких, что

$$
\left|L\left(\alpha_{k}\right)\right|>\exp \left(\sigma^{\prime \prime}\left|\alpha_{k}\right|^{\rho_{1}}\right)
$$

Теперь, используя произвол $\lambda$ в формуле (2), имеем

$$
\left|M\left(\lambda_{\nu}\right)\right|<C\left|L^{\prime}\left(\lambda_{\nu}\right)\right|\left|\alpha_{k}-\lambda_{\nu}\right| \exp \left[\left(\sigma^{\prime}-\sigma^{\prime \prime}\right)\left|\alpha_{k}\right|^{\rho_{1}}\right]
$$

откуда, считая $0<\sigma^{\prime}<\sigma^{\prime \prime}<\sigma_{1}$, при $k \rightarrow \infty$ получим $M\left(\lambda_{\nu}\right)=0, \nu \geqslant 1$, т.е. $M(\lambda) \equiv 0$. Следовательно, не любую функцию из $\left[\rho_{1}, \sigma_{1}\right)$ можно разложить в топологии $\left[\rho_{1}, \sigma_{1}\right)$ в ряд (1).

Таким образом, ограничения на рост разлагаемой функции и на топологию, в которой идет разложение, вызваны существом дела. 
2. Решение задачи. Сформулированную вьше задачу решает следуюшая теоремa.

Теорема. Для того чтобб $\forall M(\lambda) \in\left[\rho_{1}, \sigma_{1}\right)$ разлагалась в топологии $\left[\rho_{1}, \sigma_{1}\right]$ в ряд Лагранжа

$$
M(\lambda)=\sum_{\nu=1}^{\infty} \frac{L(\lambda)}{\lambda-\lambda_{\nu}} \frac{M\left(\lambda_{\nu}\right)}{L^{\prime}\left(\lambda_{\nu}\right)}
$$

необходимым и достаточным является удовлетворение функиии $L(\lambda)$ следующим двум условиям:

1) $\left|L^{\prime}\left(\lambda_{\nu}\right)\right|>\exp \left[\left(\sigma_{1}-\varepsilon_{1}\right)\left|\lambda_{\nu}\right|^{\rho_{1}}\right] \quad \forall \varepsilon_{1}>0, \quad \nu>\nu_{0}\left(\varepsilon_{1}\right)$;

2) существуют $0<\rho<\rho_{1}$ (хотя бы и сколь угодно малое) и последовательность $r_{k} \uparrow \infty$ такие, что

$$
\lim _{k \rightarrow \infty} r_{k}^{-\rho} \min _{|\lambda|=r_{k}} \ln |L(\lambda)|>0
$$

ДокАЗАтЕЛЬСтво. Необходимость. Так как $\forall M(\lambda) \in\left[\rho_{1}, \sigma_{1}\right)$ ряд $(3)$ сходится, из (3) при $\lambda=0$ следует

$$
\left|\frac{M\left(\lambda_{\nu}\right)}{\lambda_{\nu} L^{\prime}\left(\lambda_{\nu}\right)}\right|<C, \quad C=\mathrm{const},
$$

и

$$
\left|L^{\prime}\left(\lambda_{\nu}\right)\right|>\frac{\left|M\left(\lambda_{\nu}\right)\right|}{C\left|\lambda_{\nu}\right|}
$$

Зададим $\varepsilon_{1}>0$ и используем произвол в выборе $M(\lambda)$, положив $M(\lambda)$ функцией Миттаг-Леффлера

$$
M(\lambda)=E_{\rho_{1}}\left(\sigma_{1}^{\prime 1 / \rho_{1}} \lambda\right)=\sum_{n=0}^{\infty} \frac{\left(\sigma^{1 / \rho_{1}} \lambda\right)^{n}}{\Gamma\left(n / \rho_{1}+1\right)}, \quad \sigma_{1}^{\prime}=\sigma_{1}-\frac{\varepsilon_{1}}{3} .
$$

Учитьвая асимптотику этой функции, можно утверждать, что в некотором угле $\Delta_{1}=$ $\{\lambda:|\arg \lambda| \leqslant \alpha\}$, достаточно малого раствора,

$$
|M(\lambda)|>\exp \left[\left(\sigma_{1}^{\prime}-\frac{\varepsilon_{1}}{3}\right)|\lambda|^{\rho_{1}}\right], \quad|\lambda|>r_{1}\left(\varepsilon_{1}\right) .
$$

В точках $\lambda_{\nu}$, попавших в $\Delta_{1}$, при больших $\nu$

$$
\left|L^{\prime}\left(\lambda_{\nu}\right)\right|>\frac{1}{C\left(\lambda_{\nu}\right)} \exp \left[\left(\sigma_{1}^{\prime}-\frac{\varepsilon_{1}}{3}\right)\left|\lambda_{\nu}\right|^{\rho_{1}}\right]
$$

Поворачивая $\Delta_{1}$ на $\alpha$ и выбирая в качестве $M(\lambda)$ функцию $E_{\rho_{1}}\left(\sigma_{1}^{\prime 1 / \rho_{1}} \lambda e^{-i \alpha}\right)$, получим угол $\Delta_{2}$ такой, что при $\lambda_{\nu}$ попавших в $\Delta_{2}$, продолжает действовать оценка (6). И так далее. Через конечное число шагов исчерпаем всю плоскость и получим при больших $\nu$ неравенство

$$
\left|L^{\prime}\left(\lambda_{\nu}\right)\right|>\exp \left[\left(\sigma_{1}-\varepsilon_{1}\right)\left|\lambda_{\nu}\right|^{\rho_{1}}\right]
$$

т.е. оценку (4). 
Далее, исходя из равенства (3) и используя только что полученную оценку, вне кружков

$$
A_{\nu}: \quad\left|\lambda-\lambda_{\nu}\right|<\left|\lambda_{\nu}\right|^{-h}, \quad h>\rho_{1},
$$

имеем

$$
\left|\frac{M(\lambda)}{L(\lambda)}\right|<\sum_{\nu=1}^{\infty}\left|\lambda_{\nu}\right|^{h} \frac{\left|M\left(\lambda_{\nu}\right)\right|}{\left|L^{\prime}\left(\lambda_{\nu}\right)\right|}<C \sum_{\nu=1}^{\infty} \frac{\exp \left[\left(\sigma_{1}-\varepsilon\right)\left|\lambda_{\nu}\right|^{\rho_{1}}\right]}{\exp \left[\left(\sigma_{1}-\varepsilon_{1}\right)\left|\lambda_{\nu}\right|^{\left.\rho_{1}\right]}\right.} .
$$

Выбирая $\varepsilon>0$ так, что $\varepsilon_{1}<\varepsilon$, получим

$$
|L(\lambda)|>C_{1}|M(\lambda)|, \quad \lambda \notin A_{\nu}
$$

Заметим, что сумма радиусов $A_{\nu}$ - конечная, ибо $h>\rho_{1}$ и $\sum\left|\lambda_{\nu}\right|^{-h}<\infty$.

Как и прежде, распоряжаясь выбором $M(\lambda)$, используя функцию Миттаг-Леффлера и углы $\Delta_{1}, \Delta_{2}, \ldots$, в итоге $\forall \varepsilon_{0}>0$ получим оценку

$$
|L(\lambda)|>\exp \left[\left(\sigma_{1}-\varepsilon_{0}\right)|\lambda|^{\rho_{1}}\right], \quad|\lambda|>r_{0}\left(\varepsilon_{0}\right), \quad \lambda \notin A_{\nu}
$$

Пусть сумма диаметров $A_{\nu}$ равна $H$. Повернем центры $A_{\nu}$ по соответствующим окружностям до попадания на положительную полуось. Повернутые кружки пересекутся с полуосью по некоторому множеству $K$. Ясно, что на $[0,2 H]$ найдется $r_{1} \notin K$, на $[2 H, 4 H]$ найдется $r_{2} \notin K$ и т.д. Таким образом, имеются $r_{k} \uparrow \infty$ такие, что окружности $|\lambda|=r_{k}$ проходят вне кружков $A_{\nu}$ и на них справедлива оценка (7).

Полагая $\rho=\rho_{1} / 2$, из (7) имеем

$$
r_{k}^{-\rho} \min _{|\lambda|=r_{k}} \ln |L(\lambda)|>\left(\sigma_{1}-\varepsilon_{0}\right) r_{k}^{\rho_{1}-\rho} \rightarrow+\infty,
$$

т.е. справедливо соотношение (5).

Достаточность. Заметим, что условие (4) влечет сходимость ряда (3) в топологии $\left[\rho_{1}, \sigma_{1}\right] \forall M(\lambda) \in\left[\rho_{1}, \sigma_{1}\right)$. Действительно, в книге [2] приведена равномерная по $\nu$ оценка

$$
\left|\frac{L(\lambda)}{\lambda-\lambda_{\nu}}\right|<\exp \left[\left(\sigma_{1}+\varepsilon\right)|\lambda|^{\rho_{1}}\right], \quad|\lambda|>r_{0}(\varepsilon) .
$$

Тогда при больших $\nu$

$$
\left|\frac{L(\lambda)}{\lambda-\lambda_{\nu}} \frac{M\left(\lambda_{\nu}\right)}{L^{\prime}\left(\lambda_{\nu}\right)}\right|<e^{\left(\sigma_{1}+\varepsilon\right)|\lambda|^{\rho_{1}}} \frac{\exp \left[\left(\sigma_{1}-2 \varepsilon_{1}\right)\left|\lambda_{\nu}\right|^{\rho_{1}}\right]}{\exp \left[\left(\sigma_{1}-\varepsilon_{1}\right)\left|\lambda_{\nu}\right|^{\rho_{1}}\right]},
$$

и члены ряда (3) допускают оценку

$$
\left|\frac{L(\lambda)}{\lambda-\lambda_{\nu}} \frac{M\left(\lambda_{\nu}\right)}{L^{\prime}\left(\lambda_{\nu}\right)}\right|<C e^{-\varepsilon_{1}\left|\lambda_{\nu}\right|^{\rho_{1}}} \exp \left[\left(\sigma_{1}+\varepsilon\right)\left|\lambda_{\nu}\right|^{\rho_{1}}\right],
$$

откуда и следует сходимость ряда $(3)$ в $\left[\rho_{1}, \sigma_{1}\right]$.

Заметим, что, по сути дела, доказано такое утверждение: для того, чтобы $\forall M(\lambda) \in$ $\left[\rho_{1}, \sigma_{1}\right)$ ее ряд $(3)$ сходился в $\left[\rho_{1}, \sigma_{1}\right]$ (вопрос о сумме ряда открыт), необходимо и достаточно условие (4). 
Далее, отправляясь от $\rho$, фигурировавшего в (5), и выбирая $\sigma \neq 0, \infty$, введем в рассмотрение целую функцию порядка $\rho$ и типа $\sigma$

$$
f(z)=1+\sum_{n=1}^{\infty}(\sigma e \rho)^{n / \rho} \frac{z^{n}}{n^{n / \rho}}=\sum_{n=0}^{\infty} a_{n} z^{n} .
$$

Вычислим $\rho_{2}$ и $\sigma_{2}$ из равенств

$$
\frac{1}{\rho_{1}}+\frac{1}{\rho_{2}}=\frac{1}{\rho}, \quad\left(\sigma_{1}, \rho_{1}\right)^{1 / \rho_{1}}\left(\sigma_{2} \rho_{2}\right)^{1 / \rho_{2}}=(\sigma \rho)^{1 / \rho} .
$$

Если

$$
M(\lambda)=\sum_{n=0}^{\infty} b_{n} \lambda^{n} \in\left[\rho_{1}, \sigma_{1}\right) \quad \text { и } \quad F(z)=\sum_{n=0}^{\infty} d_{n} a_{n} z^{n} \in\left[\rho_{2}, \sigma_{2}\right]
$$

то, как показано в [3], выражение

$$
\sum_{0}^{\infty} b_{n} d_{n}
$$

имеет смысл (ряд абсолютно сходится) и, более того, представляет собой линейньй непрерьвньй функционал $S_{M}(F)$, действующий в $\left[\rho_{2}, \sigma_{2}\right]$. Ясно, что $\rho_{2}>\rho$ и $\forall \lambda \in \mathbb{C}$ функция $f(\lambda z)$ содержится в $\left[\rho_{2}, \sigma_{2}\right]$, причем

$$
S_{M}[f(\lambda z)]=\sum_{0}^{\infty} b_{n} \lambda^{n}=M(\lambda)
$$

Когда $|\lambda|<r_{k}$

$$
\Phi_{r_{k}}(z, \lambda)=f(\lambda z)-\sum_{\left|\lambda_{\nu}\right|<r_{k}} \frac{L(\lambda)}{\lambda-\lambda_{\nu}} \frac{f\left(\lambda_{\nu} z\right)}{L^{\prime}\left(\lambda_{\nu}\right)}=\frac{L(\lambda)}{2 \pi i} \int_{|\mu|=r_{k}} \frac{f(\mu z) d \mu}{(\mu-\lambda) L(\mu)}
$$

При любом фиксированном $\lambda$ и больших $k|\mu-\lambda|>1$ и

$$
\left|\Phi_{r_{k}}(z, \lambda)\right| \leqslant C r_{k} \max _{|\mu|=r_{k}}\left|\frac{f(\mu z)}{L(\mu)}\right|<\frac{\exp \left[(\sigma+\varepsilon)|z|^{\rho} r_{k}^{\rho}\right]}{\exp \left(A r_{k}^{\rho}\right)}
$$

где $A>0, k \rightarrow \infty$. Отсюда следует, что равномерно в окрестности $z=0$

$$
f(\lambda z)=\lim _{k \rightarrow \infty} \sum_{\left|\lambda_{\nu}\right|<r_{k}} \frac{L(\lambda)}{\lambda-\lambda_{\nu}} \frac{f\left(\lambda_{\nu} z\right)}{L^{\prime}\left(\lambda_{\nu}\right)}
$$

Докажем, что на самом деле $\forall \lambda \in \mathbb{C}$ по $z$ в топологии $\left[\rho_{2}, \sigma_{2}\right]$ справедлива формула

$$
f(\lambda z)=\sum_{\nu=1}^{\infty} \frac{L(\lambda)}{\lambda-\lambda_{\nu}} \frac{f\left(\lambda_{\nu} z\right)}{L^{\prime}\left(\lambda_{\nu}\right)}
$$


При любом фиксированном $\lambda$ и больших $\nu$

$$
\left|\frac{L(\lambda)}{\lambda-\lambda_{\nu}} \frac{f\left(\lambda_{\nu} z\right)}{L^{\prime}\left(\lambda_{\nu}\right)}\right|<C e^{-\varepsilon_{0}\left|\lambda_{\nu}\right|^{\rho_{1}}} \exp \left[(\sigma+\varepsilon)\left|\lambda_{\nu}\right|^{\rho}|z|^{\rho}-\left(\sigma_{1}-2 \varepsilon_{0}\right)\left|\lambda_{\nu}\right|^{\rho_{1}}\right] .
$$

Положим $\varepsilon_{1}=2 \varepsilon_{0}$ и найдем

$$
A=\max _{x} \exp \left[(\sigma+\varepsilon) x^{\rho}|z|^{\rho}-\left(\sigma_{1}-\varepsilon_{1}\right) x^{\rho_{1}}\right] .
$$

Максимум достигается при

$$
x=\left[\frac{(\sigma+\varepsilon) \rho|z|^{\rho}}{\left(\sigma_{1}-\varepsilon_{1}\right) \rho_{1}}\right]^{1 /\left(\rho_{1}-\rho\right)},
$$

откуда, используя формулы (8),

$$
\begin{aligned}
A & =\exp \left\{|z|^{\rho_{2}} \frac{(\sigma+\varepsilon)^{\rho_{2} / \rho}}{\left(\sigma_{1}-\varepsilon_{1}\right)^{\rho_{2} / \rho_{1}}}\left[\left(\frac{\rho}{\rho_{1}}\right)^{\rho_{2} / \rho_{1}}-\left(\frac{\rho}{\rho_{1}}\right)^{\rho_{2} / \rho}\right]\right\} \\
& =\exp \left\{|z|^{\rho_{2}} \frac{1}{\rho_{2}} \frac{[(\sigma+\varepsilon) \rho]^{\rho_{2} / \rho}}{\left[\left(\sigma_{1}-\varepsilon_{1}\right) \rho_{1}\right]^{\rho_{2} / \rho_{1}}}\right\}=\exp \left[\left(\sigma_{2}+\varepsilon_{2}\right)|z|^{\rho_{2}}\right]
\end{aligned}
$$

где $\varepsilon_{2}>0$ - мало, вместе с $\varepsilon>0$ и $\varepsilon_{1}>0$.

Но тогда из оценки (12) следует, что ряд (11) сходится в $\left[\rho_{2}, \sigma_{2}\right]$. Объединяя этот факт с (10), получим, что в $\left[\rho_{2}, \sigma_{2}\right]$ справедлива формула (11).

Возьмем $\forall M(\lambda) \in\left[\rho_{1}, \sigma_{1}\right)$ и подействуем по $z$ функционалом $S_{M}$ на обе части (11). Почленное применение функционала законно, и в соответствии с (9) получим $\forall \lambda \in \mathbb{C}$

$$
M(\lambda)=\sum_{\nu=1}^{\infty} \frac{L(\lambda)}{\lambda-\lambda_{\nu}} \frac{M\left(\lambda_{\nu}\right)}{L^{\prime}\left(\lambda_{\nu}\right)}
$$

Сходимость последнего ряда в $\left[\rho_{1}, \sigma_{1}\right]$ уже была установлена.

Московский энергетический институт

\section{СПИСОК ЦИТИРОВАННОЙ ЛИТЕРАТУРЫ}

[1] Ибрагимов И. И. Методы интерполяции функций и некоторые их применения. М.: Наука, 1971.

[2] Леонтьев А. Ф. Ряды экспонент. М.: Наука, 1976.

[3] Фролов Ю. Н. Об аппроксимации решений уравнения бесконечного порядка в обобщенных производных посредством элементарных решений // Исследования по теории аппроксимации функций. Уфа, 1979. С. 268-281. 